\title{
Generation of Nyquist sinc pulses using intensity modulators
}

\author{
Marcelo A. Soto ${ }^{1, *}$, Mehdi Alem ${ }^{1}$, Mohammad Amin Shoaie², Armand Vedadi' ${ }^{2}$, Camille-Sophie Brès², \\ Luc Thévenaz ${ }^{1}$, and Thomas Schneider ${ }^{3}$ \\ ${ }^{1}$ EPFL Swiss Federal Institute of Technology, Group for Fibre Optics (GFO), SCI-STI-LT, Station 11, CH-1015 Lausanne, Switzerland \\ ${ }^{2}$ EPFL Swiss Federal Institute of Technology, Photonic Systems Laboratory (PHOSL), SCI-IEL, Station 11, CH-1015 Lausanne, Switzerland \\ ${ }^{3}$ Institut für Hochfrequenztechnik, Hochschule für Telekommunikation Leipzig, Gustav-Freytag-Str. 43-45, 04277 Leipzig, Germany \\ *Email: marcelo.soto@epfl.ch
}

\begin{abstract}
Optical sinc-shaped Nyquist pulses are produced based on the generation of an ideal frequency comb using cascaded intensity modulators. Nyquist pulses with 9.8-ps temporal width, 82-fs jitter and more than $40 \mathrm{~dB}$ SNR are achieved.

OCIS codes: (060.4510) Optical communications; (060.4080) Modulation; (320.5550) Pulses.
\end{abstract}

\section{Introduction}

The increasing demand for higher data transmission rates during the last decade has motivated scientists to search for new approaches to improve the spectral efficiency of optical communication networks. In particular, using orthogonal frequency division multiplexing (OFDM) or Nyquist pulses [1], ultrahigh data rate and ultrahigh spectral efficiency can be achieved simultaneously. In OFDM, rectangular pulses in time domain are used, leading to sincshaped frequency bands; while in Nyquist pulse modulation the use of sinc-shaped temporal pulses leads to a rectangular spectrum for an optimal usage of the available bandwidth [1].

Nyquist pulses can be orthogonally multiplexed in a wavelength division multiplexing (WDM) scheme so-called Nyquist-WDM [2], or in a time division multiplexing (TDM) scheme so-called orthogonal TDM [3]. Nyquist pulses have interesting properties in terms of orthogonality [1-4], leading to zero inter-symbol interference (ISI) despite the large pulse overlap in time domain [4]. The spectral efficiency of Nyquist pulses can also increase considerably the tolerance against dispersion and nonlinearities, even when ultrashort pulses are used [5-6]. Recently, a 32.5 Tbit/s Nyquist WDM transmission with a spectral efficiency of $6.4 \mathrm{bit} / \mathrm{s} / \mathrm{Hz}$ was reported [2]. Many different approaches have been proposed to Nyquist pulses [2,3,7]. However, besides the high complexity of the proposed methods, they cannot generate ideal sinc pulses, leading to inter-channel crosstalk and to an inefficient use of the spectrum.

In this paper a method to produce perfect sinc-shaped Nyquist pulses is proposed based on the generation of a frequency comb with an ideal rectangular spectrum, within the best experimental tolerance. The effectiveness of the method is demonstrated using two cascaded electro-optic modulators (EOM), achieving highly-stable sinc pulses.

\section{Principle and experimental validation}

When light is intensity modulated using a properly-adjusted EOM bias voltage, three equally-spaced spectral components (carrier and two sidebands) with the same amplitude and phase can be obtained. Thus, a flat frequency comb with many phase-locked lines can be generated combining EOMs in cascade, each of them driven with a different modulating frequency. If higher-order sidebands can be highly suppressed, a frequency comb with a perfect rectangular shape will be produced; this in turn corresponds to ideal Nyquist sinc pulses in the time domain.

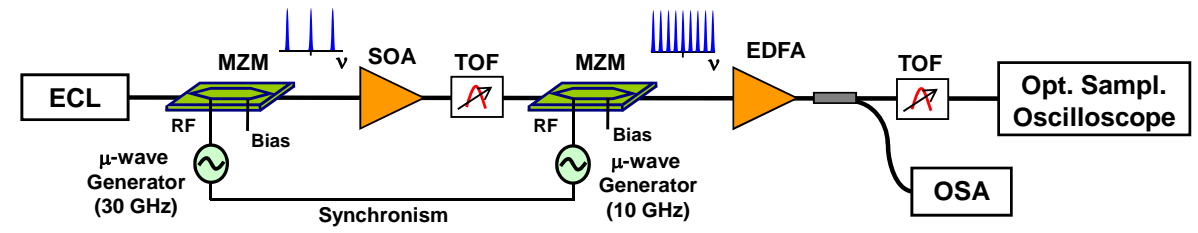

Fig. 1. Experimental setup for sinc-shaped Nyquist pulse generation based on an ideal phase-locked, flat frequency comb.

Fig. 1 shows the experimental setup used to generate a flat frequency comb with 9 phase-locked and equally spaced spectral lines. Two cascaded Mach-Zehnder modulators (MZMs) and two synchronized microwave generators are used. The first MZM is driven by an RF signal at $30 \mathrm{GHz}$, while the second one is driven at $10 \mathrm{GHz}$. The phase difference of the RF signals is adjusted to ensure that the generated lines have the same phase. Another alternative to drive both MZMs is to use only one generator together with a frequency tripler and a phase shifter. A semiconductor optical amplifier (SOA) and an Erbium-doped fiber amplifier (EDFA) are used, together with two tunable optical filters (TOF), to measure pulses on a 500-GHz optical sampling oscilloscope. Note that the optical filters are used only to reduce amplified spontaneous emission noise and are not required for spectral shaping.

Defining $\epsilon=V_{B} / V_{\pi}$ and $\alpha=v_{s} / V_{\pi}$, in which $V_{\pi}, V_{B}$ and $v_{s} \cos \left(\omega_{s} t\right)$ are the half-wave voltage, DC bias voltage and 
RF signal applied to an MZM, the output field of the MZM can be expressed as [8]:

$$
E(t)=\sum_{k=-\infty}^{+\infty}(-1)^{k}\left\{\cos (\pi \epsilon) J_{2 k}(\pi \alpha) \cos \left(\omega t+2 k \omega_{s} t\right)+\sin (\pi \epsilon) J_{2 k-1}(\pi \alpha) \cos \left(\omega t+(2 k-1) \omega_{s} t\right)\right\},
$$

where $J_{k}$ is the first kind Bessel function of order $k$. As described in Eq. (1), by adjusting the RF driving voltage $\alpha$ and the DC bias $\epsilon$ the amplitude of the carrier and sidebands can be changed. Fig. 2 shows a contour plot with the amplitude difference between the 1st-order sidebands and the carrier (i.e. $J_{1}(\pi \alpha) \sin (\pi \varepsilon)-J_{0}(\pi \alpha) \cos (\pi \varepsilon)$ ) as a function of the normalized voltages $\alpha$ and $\epsilon$. It can be seen that there are many combinations of $\alpha$ and $\epsilon$ in which those amplitudes are the same (represented by the thick straight lines at zero level). From Eq. (1), the relation between $\alpha$ and $\epsilon$ that ensures the same amplitude for the carrier and 1st-order sidebands can be calculated as:

$$
\epsilon=\pi^{-1} \tan ^{-1}\left[-J_{0}(\pi \alpha) / J_{1}(\pi \alpha)\right] \text {. }
$$

Based on Eq. (1), Fig. 3 shows the amplitude of the carrier and the 1st-order sidebands (right vertical axis) as a function of $\alpha$ when $\epsilon$ is adjusted according to Eq. (2). The figure also shows the power difference of the 2nd- and 3rd-order sidebands with respect to the carrier power as a function of $\alpha$. It can be seen that when $\alpha=0.4$, the maximum attainable amplitude of the carrier and 1st-order sidebands can be reached with $15 \mathrm{~dB}$ suppression of the 2nd-order sidebands. However, greater higher-order sideband suppression can still be achieved by reducing $\alpha$ (slightly decreasing the total power).

Fig. 4(a) shows the generated phase-locked frequency comb. The power variation among lines was measured to be below $0.2 \mathrm{~dB}$, while higher-order sidebands are suppressed by $27 \mathrm{~dB}$. Fig. 4(b) shows the acquired Nyquist pulses (straight blue line) in timedomain, together with the theoretical sinc curve (dashed red line). We can observe that the generated sinc pulse sequence perfectly fits to the expected theoretical shape (with a power difference lower than 1\%), exhibiting a full-width at half maximum (FWHM) of $9.8 \mathrm{ps}$, and a repetition rate of $100 \mathrm{ps}$. Fig. 4(c) shows a color-grade plot of one of the generated sinc-shaped pulses. The stability of the generated signal was evaluated in terms of rms jitter and SNR, which were measured as $82 \mathrm{fs}$ and more than $40 \mathrm{~dB}$, respectively.

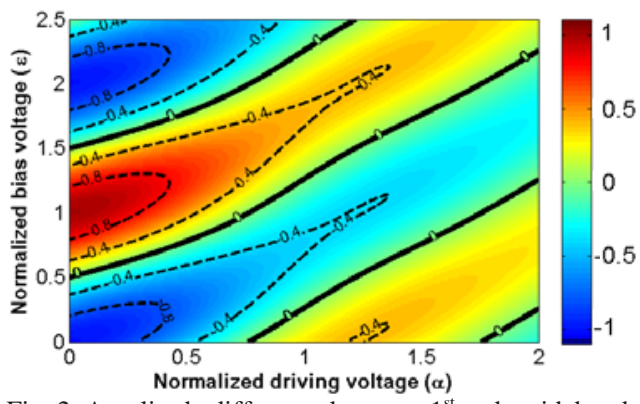

Fig. 2. Amplitude difference between $1^{\text {st }}$-order sidebands and carrier $\left[J_{1}(\pi \alpha) \sin (\pi \varepsilon)-J_{0}(\pi \alpha) \cos (\pi \varepsilon)\right]$.

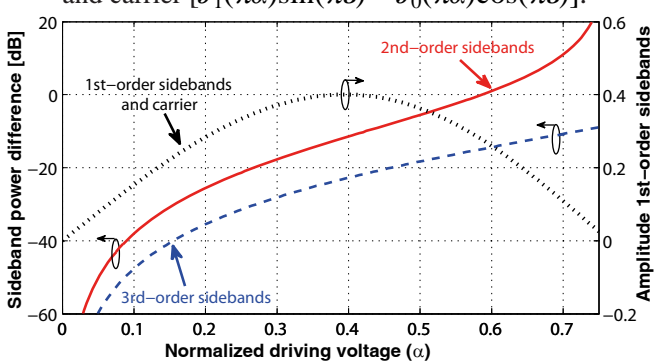

Fig. 3. $1^{\text {st }}$-order sideband amplitude (right axis) and power difference (in $\mathrm{dB}$ ) between $2^{\text {nd }}$ and $3^{\text {rd }}$ order sidebands with respect to $1^{\text {st }}$ order sidebands (left axis).
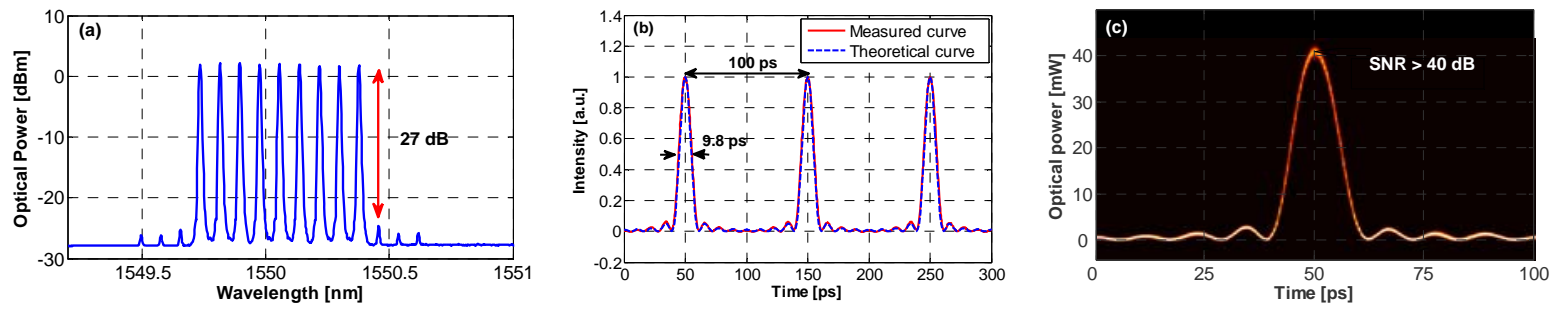

Fig. 4. (a) Optical spectrum and (b) time waveform of the generated Nyquist pulses. The acquired sinc-shaped pulse sequence is compared in (b) with the theoretical periodic sinc funtion described in Eq. (2) showing perfect fitting. (c) Color-grade figure indicating high SNR ( $>40 \mathrm{~dB}$ ).

In conclusion, we have shown a simple method to produce ideal, low-noise and highly stable sinc-shaped Nyquist pulses using electro-optic intensity modulators. The technique offers the possibility to easily change the pulse parameters, and can be straightforwardly incorporated in the modulation stage of telecommunications systems in combination to higher-order modulation formats. Furthermore, the method can also provide great benefits to improve the performance of all-optical sampling devices and photonic analog-to-digital converters (ADC).

\section{References}

[1] R. Schmogrow et al., "Real-time Nyquist pulse generation beyond $100 \mathrm{Gbit} / \mathrm{s}$ and its relation to OFDM,” OpEx. 20, 317-337 (2012).

[2] D. Hillerkuss et. al., "Single-Laser 32.5 Tbit/s Nyquist WDM Transmission,” Opt. Commun. Netw. 4, 715-723 (2012).

[3] M. Nakazawa et al., "Ultrahigh-speed “orthogonal” TDM transmission with an optical Nyquist pulse train,” OpEx. 20, 1129-1140 (2012).

[4] H. Nyquist, "Certain topics in telegraph transmission theory,” Trans. Am. Inst. Electr. Eng. 47, 617-644 (1928).

[5] T. Hirooka et al., "Highly dispersion-tolerant 160 Gbaud optical Nyquist pulse TDM transmission over 525 km," OpEx. 20, 15001-15007 (2012).

[6] T. Hirooka et. al., "Linear and nonlinear propagation of optical Nyquist pulses in fibers," OpEx. 20, 19836-19849 (2012).

[7] R. Schmogrow et al., "512QAM Nyquist sinc-pulse transmission at $54 \mathrm{Gbit} / \mathrm{s}$ in an optical bandwidth of $3 \mathrm{GHz}$," OpEx. 20, $6439-6447$ (2012).

[8] Q. Chang et al., "Generation of optical comb frequency signal with high spectral flatness using two cascaded optical modulators," in Optical

Fiber Communication \& Optoelectronic Exposition \& Conference, 2008. AOE 2008. Asia (2008), paper SaB4. 